\title{
The combined role of MR spectroscopy and perfusion imaging in preoperative differentiation between high- and low- grade gliomas
}

Abdel-Monem S. Hasan ${ }^{1 *}$ (D, Abdel Karem Hasan ${ }^{1}$, Hasan I. Megally' ${ }^{1}$ Mohammed Khallaf ${ }^{2}$ and Abolhasan Haseib

\begin{abstract}
Background: Brain tumors are an important health problem. The preoperative classification of gliomas by noninvasive techniques is a significant problem. Relative cerebral blood volume and spectroscopy have the ability to sample the entire lesion non-invasively. The present study aims to evaluate the combined role of dynamic susceptibility perfusion and spectroscopy in the classification of primary brain tumors. The combination of both provides overall diagnostic accuracy (100\%). Relative cerebral blood volume in peritumoral region plays an important additional role in this regard.
\end{abstract}

Results: On the basis of histopathology, among 50 patients with brain tumors, high-grade gliomas accounted for $58 \%$, while low-grade gliomas accounted for $42 \%$. The relative cerebral blood volume in the tumor had the best sensitivity, specificity, and accuracy of $96.8 \%, 95.3 \%$, and 96 , respectively. The use of relative cerebral blood volume and choline/N-acetyl Aspartate increased diagnostic accuracy by $100 \%$.

Conclusion: The combination of magnetic resonance spectroscopy and perfusion can increase sensitivity and positive predictive value to define the degree of glioma.

Keywords: Dynamic susceptibility MRI, Glioma grading, MRI perfusion, MR spectroscopy, Relative cerebral blood volume

\section{Background}

It is very difficult to differentiate an isolated intra-axial brain tumor and decide whether it is a primary or metastatic tumor, low grade or high grade. Although some conventional magnetic resonance imaging results are characteristic, it is difficult, sometimes impossible, to differentiate these tumors and in such cases; overall tumor resection or stereotactic biopsy are required for an accurate diagnosis. Another problem is the preoperative classification of glial tumors by non-invasive techniques [1-4].

Advanced neuroimaging (magnetic resonance spectroscopy and perfusion) combines the spatial localization capabilities of magnetic resonance imaging with biochemical and

\footnotetext{
* Correspondence: dr_abdelmonem84@yahoo.com

'Department of Diagnostic and Interventional Radiology, Faculty of

Medicine, Assiut University, Assiut 71116, Egypt

Full list of author information is available at the end of the article
}

functional tissue information, which has been shown to discriminate tumor grades by describing the metabolic changes reflecting cell density, anaplasia, and mitotic index [5].

Spectroscopic MRI (MRS) finding provides metabolic information supplementing the anatomical information obtained with conventional MRI [6].

Relative cerebral blood volume ( $\mathrm{rCBV}$ ) mirrors the vascular multiplication; in this way, the level of the neovascularization and can be obtained from dynamic susceptibility contrast magnetic resonance perfusion imaging (DSC-MRI) [7-10].

It is known that cell permeation usually associated with peritumoral neovascularization which is a specific character for a highly malignant brain tumor [11]; this finding has been reported many times in different studies aiming to differentiate high-grade glioma (HGG) from metastases [12-14]. Such a feature may be helpful 
in glioma grading because it is seen in high-grade glioma and not in low-grade glioma [15-17].

\section{Methods}

Our study composed of 50 cases (24 females and 26 male) with gliomas. Their ages ranged from 3 to 70 years with a mean age of $38.89 \pm 21.6$ years. All patients examined by MR scanner (Philips Achieva, 1.5 Tesla):

I. Conventional MRI

a. Screening the patient for any contraindications to the MRI examination, such as cardiac pacemakers or aneurysmal clips.

b. Sedation provided for irritable patients and pediatrics.

c. The standard circularly polarized head coil used as the receiver coil.

d. The following sequences used in conventional MR examination:

1. Axial, coronal T2-weighted turbo spin-echo (T2 TSE) using the following parameters: repetition time (TR) of $6000 \mathrm{~ms}$, echo time (TE) of $120 \mathrm{~ms}$, a slice thickness of $5.5 \mathrm{~mm}$, a gap of $5.5 \mathrm{~mm}, 240 \times$ 139 acquisition matrix, FOV $=230 \mathrm{~mm}$. given an acquisition time of $54 \mathrm{~s}$.

2. Axial fluid attenuation recovery (FLAIR) using the following parameters: TR of $4850 \mathrm{~ms}$, TE of 110 ms, a slice thickness of $5.5 \mathrm{~mm}$, a gap of $0 \mathrm{~mm}, 256$ $\times 160$ acquisition matrix, FOV $=230 \mathrm{~mm}$. given an acquisition time of $53.3 \mathrm{~s}$.

3. Pre and post contrast axial and sagittal T1-weighted spin echo (T1 SE) with the following parameters (post contrast sequences done after perfusion study): TR of $597 \mathrm{~ms}$, a TE of $15 \mathrm{~ms}$, a $232 \times 147$ acquisition matrix, a field of view (FOV) of 230 $\mathrm{mm}$, a slice thickness of $5.5 \mathrm{~mm}$, and a gap of 0 $\mathrm{mm}$, given an acquisition time of $1 \mathrm{~min} 29 \mathrm{~s}$. II. Advanced neuroimaging
A. MR spectroscopy

2D multi-voxel CSI with average TE echo time (144 ms) (TR $988 \mathrm{~ms}, 180 \mathrm{~mm}$ FOV, acquisition time 6 min $32 \mathrm{~s}$ ) and single Voxel 3D CSI with a voxel size of $1.5 \times 1.5 \times$ $1.5 \mathrm{~cm}^{3}$ and (2000 $144 \mathrm{~ms}$ (TR/TE) performed in each case to evaluate the levels of choline (Cho) at 3.36-3.21 ppm, creatine $(\mathrm{Cr})$ at $3.15-3.0 \mathrm{ppm}$, and $\mathrm{N}$-acetyl aspartate (NAA) at $2.18-2.01 \mathrm{ppm}$. The size and position of interest determined by examining MRI images in three dimensions (sagittal, coronal, and transverse), the goal was to include the largest part of the lesion, the peri-focal region, with the normal contralateral brain region of interest (only with evaluation by Multi-voxel 2D CSI) as much as possible and to exclude subcutaneous fat and regions with large variations in magnetic susceptibility. A proper automatic adjustment and water suppression using chemical shift selective saturation (CHESS) was performed. The ratio of metabolites: $\mathrm{Cho} / \mathrm{Cr}$ and Cho/NAA calculated by dividing the integral values of the metabolite in the same spectrum and this was done automatically by the station software working.

B. Perfusion-weighted images

1. DSC perfusion sequence:

- We used the dynamic T2 *-weighted gradient echo EPI sequence (TR 1524, TE 40, matrix $88 \times 87$, slice thickness $5 \mathrm{~mm}$, interval 0 , number of scans 40 acquisition voxel size $2.55 \times 2.55 \times 5 \mathrm{~mm}$ ) during the first pass of a bolus contrast injection.

- Perfusion imaging slices should have the same thickness, position, and interval as the FLAIR or T2 axial sequences to facilitate direct comparison of perfusion results with other pre-contrast sequences.

- The phase encoding direction needs to be AP to reduce susceptibility artifacts.

- A time series of fast T2-weighted images acquired when gadolinium passes through the tissues, producing a reduction in $\mathrm{T} 2$ intensity as a function of local concentration.

- Six sections obtained with no gap to cover the whole lesion which identified on the T2-weighted images. A series of 40 multi-session acquisitions acquired. The total acquisition time was 1 min $6 \mathrm{~s}$. Eight hundred images resulted. The first six acquisitions were made before the injection of the contrast agent to establish a baseline before the contrast. At the seventh acquisition, $0.2 \mathrm{mmol} / \mathrm{kg}$ bodyweight of gadolinium injected using a power injector at a flow rate of $5 \mathrm{ml} / \mathrm{s}$ through an 18- or 20-gauge intravenous catheter, immediately followed by an injection of a bolus of saline solution at the same rate for a total of $30 \mathrm{ml}$.

2. Post-processing: All data transferred to the Phillips workstation for analysis using the NeuroT2 * Perfusion software package. Spatial and temporal smoothing applied at a medium degree for improvement of the resolution and reduction of the effect of the motion artifact. We chose the variable gamma adjustment, then the arterial input function, and put a region of 
interest (ROI) on one of the middle cerebral arteries. Several curves appeared on the left, then we chose at least five curves almost typical of the normal brain curve, then proceeded. Cerebral blood volume (CBV) color maps were obtained. The maximum regional CBV calculated by placing the ROI on the area of interest in the solid area with the greatest intensity of color. Two ROIs established in the intratumoral and peritumoral regions to measure CBV values for each. These ROIs drawn on hot spot areas, that is, most hypervascular areas, as shown on the CBV color map. Only the ROI revealed the maximum value for each parameter selected to represent the intratumoral and peritumor $\mathrm{CBVs}$ (CBVt and CBVp). These ROIs should not include any intratumor vessel, hemorrhagic spot, calcification, or necrosis. Another ROI was drawn in the contralateral normal white matter at the same level. Then, the relative ratios (rCBVt and rCBVp) were calculated by dividing the maximum value of the intratumor or peritumoral region by the value of the contralateral normal white matter and expressed as a ratio of $\mathrm{rCBV}$ [ratio $=\mathrm{CBV}$ (lesion or perilesional edema) / CBV (contralateral normal white matter).

III. Histopathological examination: All examined patients underwent surgical intervention and histopathological examination of their samples. According to the WHO, brain tumors are classified into four grades (I to IV). For statistical analysis, they are reclassified as low-grade gliomas (WHO GRADE I and II) and high-grade gliomas (WHO GRADE III and IV).

IV. Statistical analysis: Data analysis was done using SPSS statistical software (version 23). The average and the mean of each parameter (Cho/ NAA, Cho/Cr, rCBVt, and rCBVp) for high- and low-grade gliomas were calculated. Evaluation of the significant statistical difference between HGG and LGG for each parameter were done by non-parametric Mann-Whitney test. A $P$ value of less than 0.05 was considered significant statistically. The identification of optimal cut-off value for each parameter was done using ROC analysis for best differentiation between LGG and HGG. Sensitivity, specificity, positive, negative predictive values (PPV and NPP), and accuracy were then resulted. The results of the combined role of MR spectroscopy and perfusion for glioma grading was performed by linear discriminant analysis.

\section{Results}

\section{Demographics}

Our study population consisted of 50 patients (24 females and 26 male) with brain gliomas. Their ages ranged from 3 to 70 years with a mean age of $38.89 \pm 21.6$ years.

\section{Final suggested radiological diagnosis}

Twenty-five patients diagnosed as HGG, 21 diagnosed as LGG, and only four diagnosed as medulloblastoma.

\section{Histopathological diagnosis}

Based on the WHO classification, histopathological evaluation of the specimens is shown in Table 1.

\section{Descriptive statistics for high- and low-grade gliomas regarding the overall rCBV ratios and spectroscopic data} Regarding rCBVt value, for high-grade glioma (Fig. 1), the mean was $3.5(1.8-6.13)$, and for low-grade glioma (Fig. 2) $1.16(0.5-1.7)(P=0.0001)$. There is statistical significance for differentiation between low- and highgrade glioma (Fig. 3) (shown in Table 2).

Regarding rCBVp value, for high-grade glioma, the mean was 2.4 (0.5-3), and for low-grade glioma 0.8 $(0.3-1)(P=0.0001)$. There is statistical significance for differentiation between low and high-grade glioma.

Regarding Cho/Cr value, for high-grade glioma, the mean was 3.5 (1.9-8.75), and for low-grade glioma 1.6 $(1.6-1.8)(P=0.0001)$. There is statistical significance for differentiation between low and high-grade glioma.

Regarding Cho/NAA, for high-grade glioma, the mean was $5.1(2-7.5)$, and for low-grade glioma $1.6(1.2-1.8)$ $(P=0.0001)$. There is statistical significance for differentiation between low and high-grade glioma.

Table 1 Detailed histopathological diagnosis of the studied cases

\begin{tabular}{lll}
\hline Pathological diagnosis & No. of cases & Valid percent \\
\hline Anaplastic astrocytoma & 5 & 10 \\
Diffuse anaplastic astrocytoma & 1 & 2 \\
Diffuse astrocytoma & 8 & 16 \\
Diffuse midline glioma $^{\text {DNET }}{ }^{\text {a }}$ & 1 & 2 \\
Dysplastic cerebellar gangliocytoma & 1 & 2 \\
Extraventricular neurocytoma $_{\text {Ganglioglioma }}$ & 1 & 2 \\
Glioblastoma multiforme & 3 & 2 \\
Gliosarcoma & 17 & 6 \\
Medulloblastoma & 1 & 34 \\
Oligodendroglioma & 4 & 2 \\
Pilocytic astrocytoma & 2 & 8 \\
Total & 5 & 4 \\
\hline
\end{tabular}

${ }^{\mathrm{a}} D N E T$ dysembryoplastic neuroepithelial tumours 


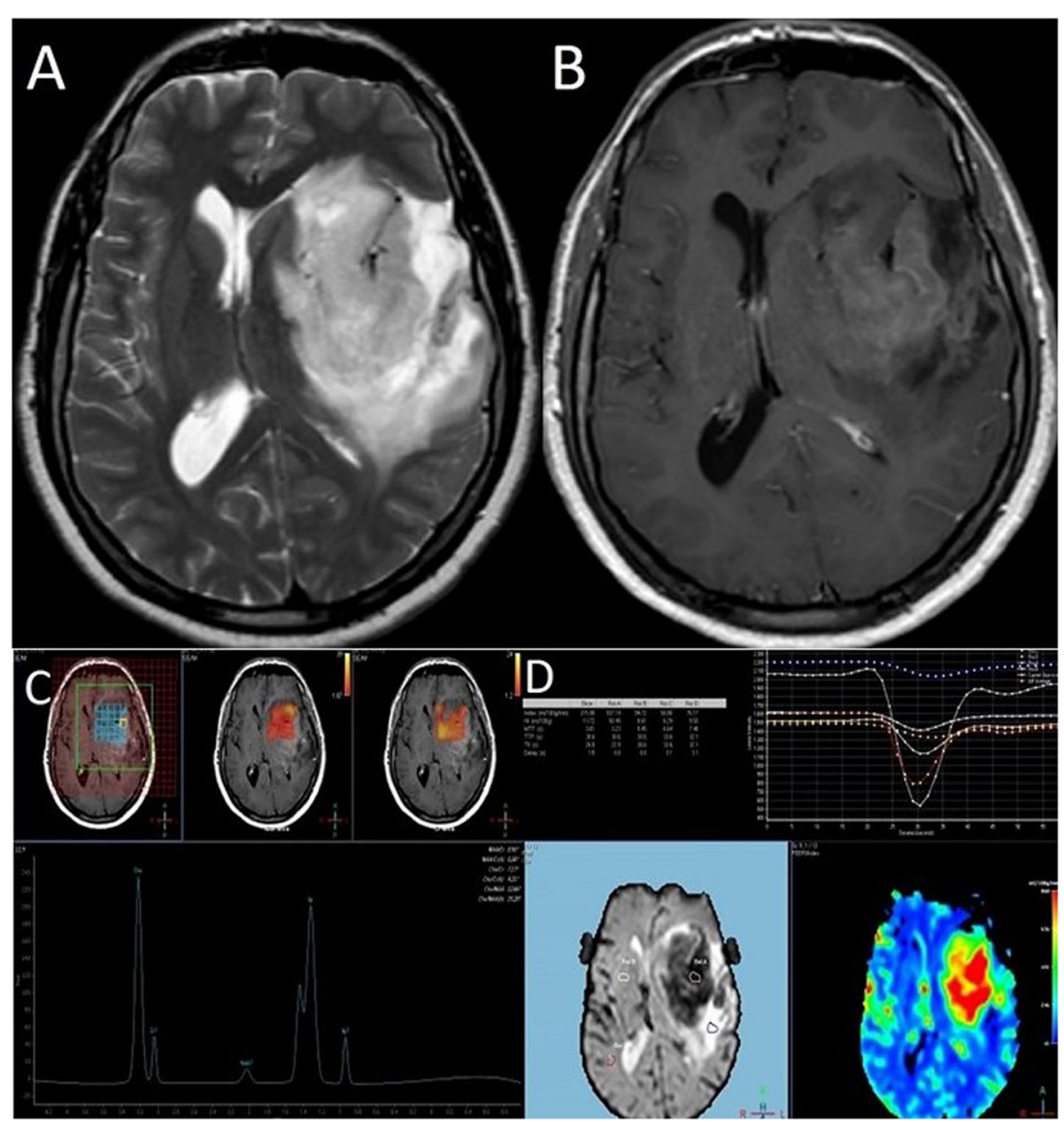

Fig. 1 A 30-year-old male presented by amnesia, slurred speech and right hemiparesis since 1 month. a Axial T2 showing left tempero-parietal large ill-defined SOL surrounded by moderate vasogenic edema. b T1 post Gd showing faint heterogeneous enhancement. c Intermediate echo spectroscopy curve shows Cho/Cr ratio 3.5, Cho/NAA ratio 6, and elevated lactate and lipid peaks. d Perfusion-weighted images PWI and rCBV color perfusion map for the lesion show marked signal drop of the lesion and area of relative hyper-perfusion rCBV5.8, and for the perilesional edema show no signal drop and area of subtle hyper-perfusion rCBV 1.2 . Collectively, the findings are in favor of HGG. Histopathological examination confirmed the diagnosis: diffuse anaplastic astrocytoma WHO GRADE III

Cut-off value, sensitivity, specificity, PPV, NPV, accuracy, and AUC (area under curve) of rCBV ratios and spectroscopic data using ROC analysis for differentiation between high and low-grade gliomas

Regarding rCBVt value, at a value more than 1.7, the sensitivity was $96.87 \%$ and specificity was 95.24\%; more than this value, it is considered HGG (Fig. 4).

Regarding rCBVp value, at a value more than 1 , the sensitivity was $87.5 \%$ and specificity was $100 \%$; more than this value, it is considered HGG (Fig. 5).

Regarding the Cho/NAA ratio, at a value more than 1.8 , the sensitivity was $100 \%$ and the specificity was $76.2 \%$; more than this value, it is considered HGG (Fig. 6) (shown in Table 3).
Regarding the Cho/Cr ratio, at a value of 1.8 , the sensitivity was $96.9 \%$ and the specificity was $76.2 \%$; more than this value, it is considered HGG.

Classification accuracy of the tumor mass with linear discriminant analysis including Cho/NAA mass plus rCBVt (leave-one-out method)

The leave-one-out method, in fact, showed that $76 \%$ of tumor masses were correctly reclassified when performing discriminant analysis regarding MRS variables alone and 100\% when regarding both MRS variables and $\mathrm{rCBV}$ (shown in Table 4).

\section{Discussion}

Our population consisted of 50 patients aged 3 to 70 years. As in the clinical setting, the incidence of HGG 


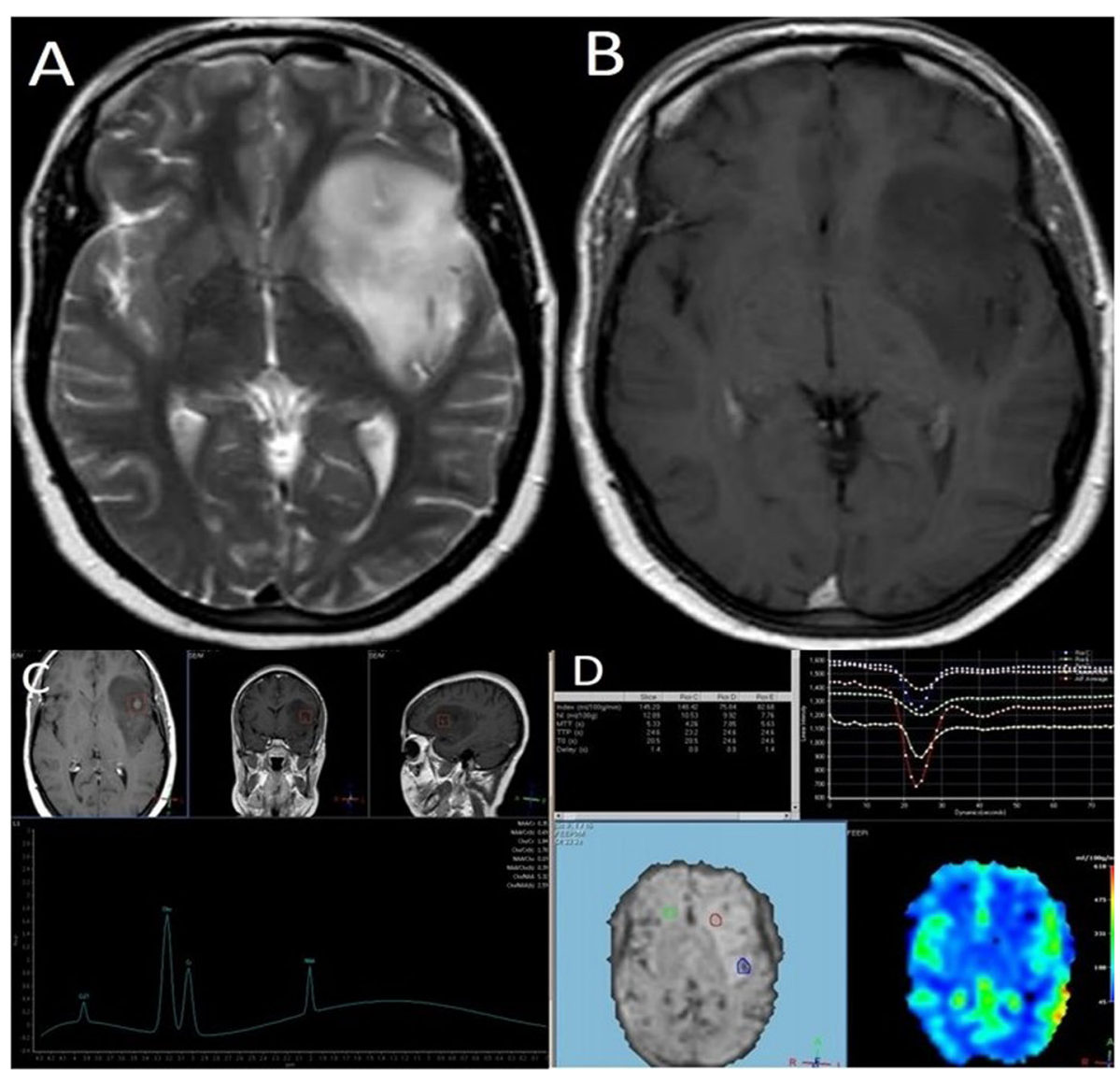

Fig. 2 A 26-year-old female presented by gradual behavior changes and right hemiparesis for 2 months. a Axial T2WI showing a large rather defined mass in left frontotemporal showing hyperintense signal, surrounded by mild vasogenic edema. b Axial T1 post Gd showing no enhancement. $\mathbf{c}$ Intermediate echo spectroscopy curve shows Cho/Cr ratio 1.5 and Cho/NAA ratio 1.8. d Perfusion-weighted images PWI and rCBV color perfusion map for the lesion and edema show a mild signal drop of the lesion and area of relative hyper-perfusion rCBV 1.5, also for edema rCBV 0.7. Collectively, the findings are in favor of LGG. Histopathological examination confirmed the diagnosis: diffuse astrocytoma WHO GRADE II

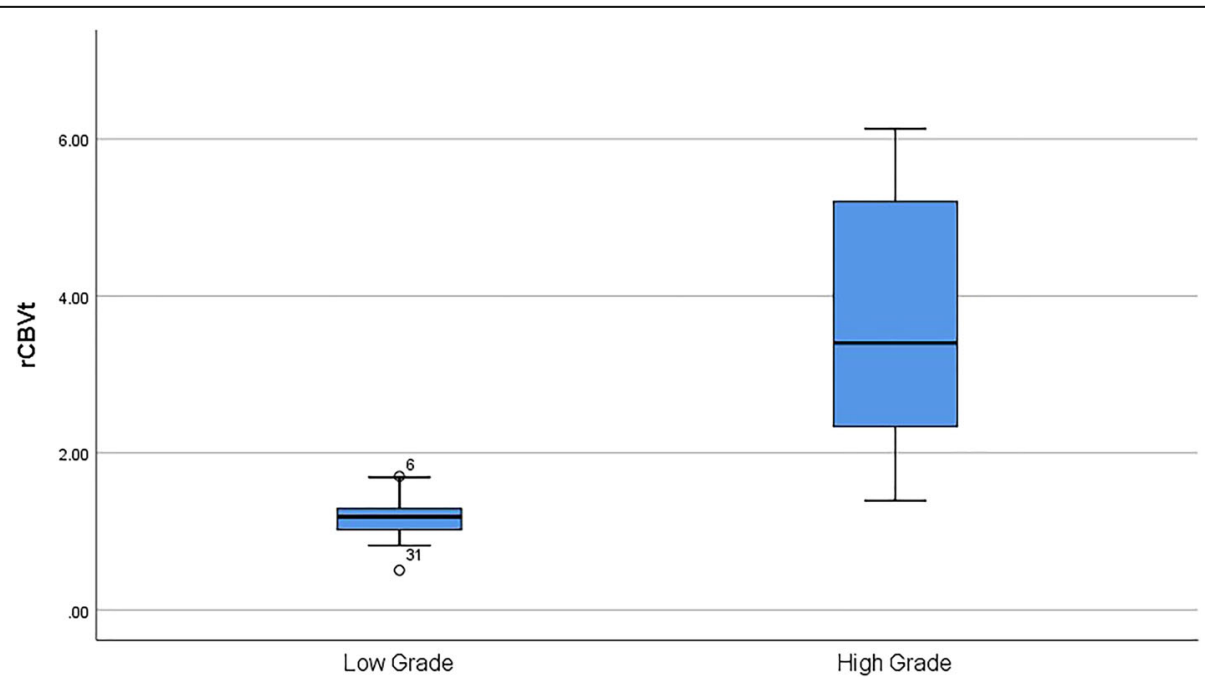

Fig. 3 Box plot for rCBVt between HG and LG 
Table 2 Descriptive statistics for high- and low-grade gliomas regarding the overall $\mathrm{rCBV}$ ratios and spectroscopic data

\begin{tabular}{llll}
\hline Variables & High grade $N=29$ & Low grade $N=21$ & $P$ value \\
\hline Age & $49(4-72)$ & $26(3-64)$ & 0.022 \\
Cho/Cr mass & $3.5(1.9-8.75)$ & $1.6(1.6-1.8)$ & 0.0001 \\
Cho/NAA mass & $5.1(2-7.5)$ & $1.6(1.2-1.8)$ & 0.0001 \\
rCBVt & $3.5(1.8-6.13)$ & $1.16(0.5-1.7)$ & 0.0001 \\
rCBVp & $2.4(0.5-3)$ & $0.8(0.29-1)$ & 0.0001 \\
\hline
\end{tabular}

(58\%) was higher than that of LGG (42\%) and it was higher for men (52\%) than for women (48\%). Glioblastoma was the commonest type of all gliomas and accounted for $34 \%$. These results are consistent with the estimated international incidence of gliomas [18-20]

At an intermediate TE, we found significant differences $(P=0.0001$ value $)$ in all reports related to the Cho metabolite (Cho/NAA and $\mathrm{Cho} / \mathrm{Cr}$ ), between low-grade and high-grade tumors (Table 2). In agreement with our results, Faten et al. [21] reported that Cho/NAA and $\mathrm{Cho} / \mathrm{Cr}$ ratios showed a statistically significant increase in high-grade astrocytoma than low-grade glioma. So MRS has superior diagnostic performance in predicting glioma grade. These findings have also been reported by Fatima et al. [22] and Naser et al. [23].

The present study revealed that for high-grade glioma, we observed $\mathrm{Cho} / \mathrm{Cr}$ ratios ranging from 1.9 to 8.75 with an average of 3.5 and Cho/NAA ratios ranging from 2 to 7.5 with an average of 5.1. With regard to the low-grade glioma, Cho/Cr ranging from 1.6 to 1.8 with an average of 1.6. Cho/NAA ratios ranging from 1.2 to 1.8 with an average ratio of 1.6 .

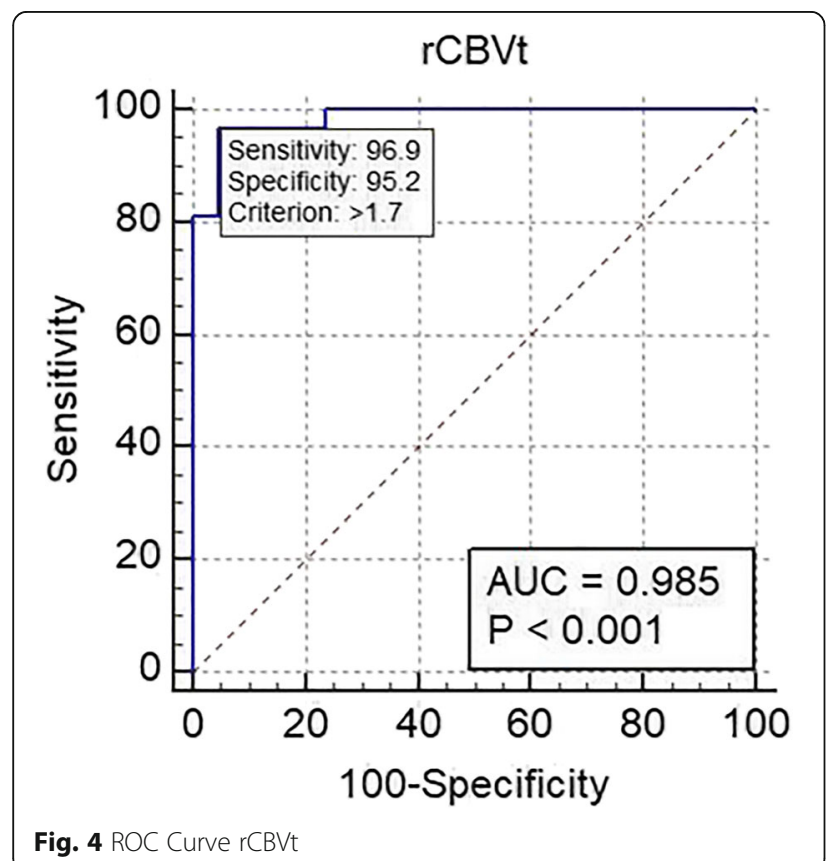

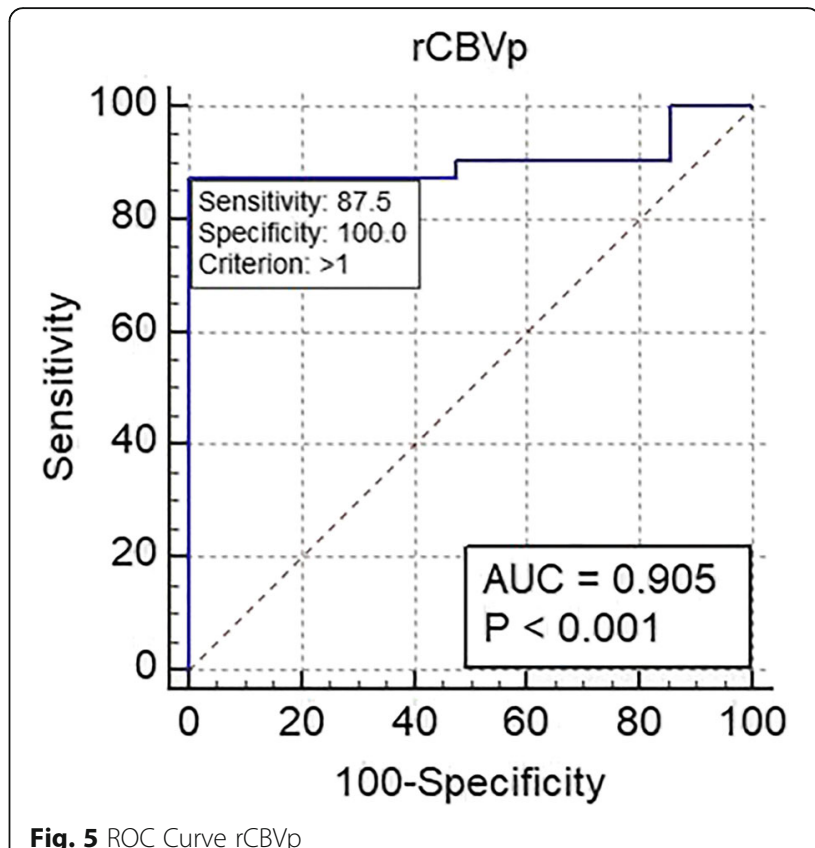

Fig. 5 ROC Curve rCBVp

Our results are in agreement with those obtained by Naser, Faten, and Fatima, who confirm that ratios related to the Cho metabolite (Cho/NAA and $\mathrm{Cho} / \mathrm{Cr}$ ) are higher for high-grade tumors than for low-grade tumors.

In our study, using ROC analysis, the cut-off values useful for intermediate TE Cho metabolite ratios were used to differentiate high-grade tumors from low-grade tumors; the Cho/NAA ratio to a cut-off value more than 1.8 and the $\mathrm{Cho} / \mathrm{Cr}$ ratio at a cut-off value of 1.7 with a higher value was considered a high-grade glioma.

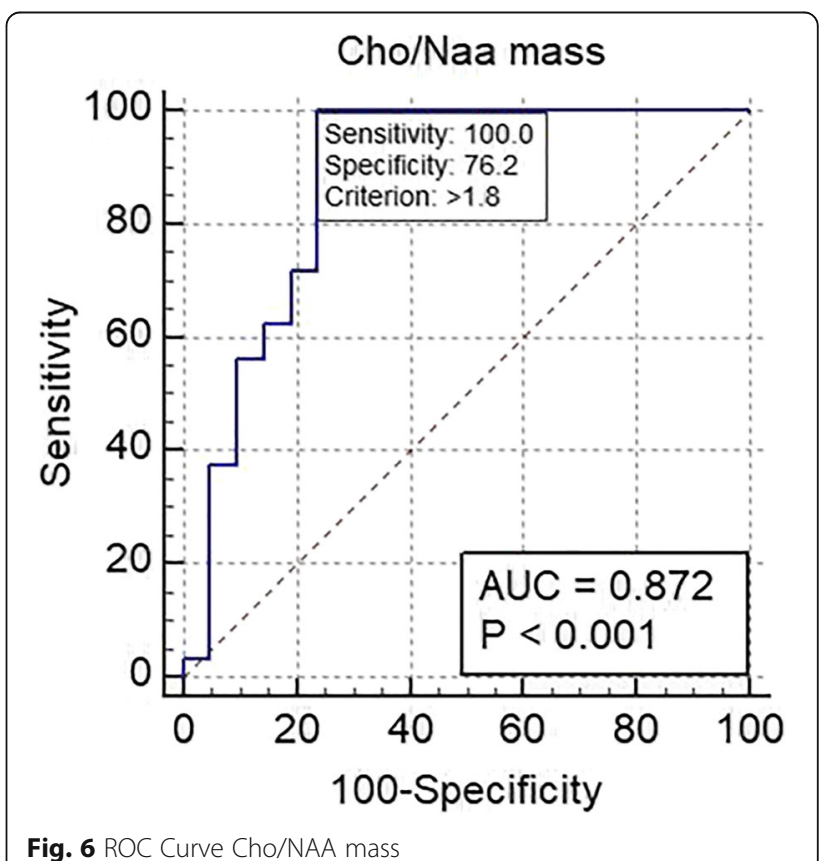


Table 3 Cut-off value, sensitivity, specificity, +PV, -PV, accuracy, and AUC of ADC values, rCBV ratios, and spectroscopic data for high- and low-grade gliomas

\begin{tabular}{llllllll}
\hline & Cut-off value & Sensitivity $\%$ & Specificity $\%$ & +PV \% & -PV \% & Accuracy \% & AUC \% \\
\hline rCBVt & More than1.7 & 96.87 & 95.24 & 96.9 & 95.2 & 96 & 98.5 \\
rCBVp & More than1 & 87.5 & 100 & 100 & 84 & 87.5 & 90.5 \\
Cho/NAAmass & More than1.8 & 100 & 76.2 & 86.5 & 100 & 76.2 & 87.2 \\
Cho/Cr mass & 1.8 & 96.9 & 76.2 & 86.1 & 94.1 & 73 & 86.5 \\
\hline
\end{tabular}

Other previous studies have also identified cut-off values [24-28]. However, cut-off values for tumor classification vary from study to study. A possible explanation for these variations could be the differences between MRS imaging methods, including MRI field strength, acquisition parameters, voxel size, and location. Another explanation behind these varieties could be the distinction made in the subject of the study, including the number of patients and the heterogeneity of the tumors.

At an intermediate TE, our results revealed that the sensitivity of Cho/NAA was $100 \%$, indicating that the HGG is correctly classified. This metabolic ratio may, therefore, be useful for determining tumor grades. The corresponding specificity of this ratio was $76.2 \%$, indicating that the less severe tumors were correctly classified. The sensitivity of the $\mathrm{Cho} / \mathrm{Cr}$ ratios was $96.9 \%$, indicating true positive high rates and false negative low rates; therefore, it is very useful for determining high-grade tumors. However, its high specificity $(76.2 \%)$ indicates that only a few low-grade tumors have been falsely identified as high grade. These results are consistent with those of Faten, Fatima, and Naser.

In this study, rCBVt was able to differentiate significantly LGG and HGG, with a $P$ value of 0.0001 . The present study showed that rCBV ratios ranged from 1.8 to 6.13 with an average of 3.5 for HGG, but for lowgrade gliomas, we found lower rCBV ratios of 0.5 at 1.7 with an average of 1.16 . This finding is in line with many previous studies $[7,9,29,30]$, highlighting the importance of rCBVt as an indicator of tumor neovascularization in glioma grading.

Table 4 Classification accuracy of the tumor mass with linear discriminant analysis including ADC value plus Cho/NAA mass plus rCBVt (leave-one-out method) (100\% of cross-validated grouped casescorrectly classified; $100 \%$ of original grouped cases correctly classified)

\begin{tabular}{ccllll}
\hline & & \multicolumn{2}{c}{ Predicted group membership } & Total \\
\cline { 3 - 4 } & & Low grade & High grade & \\
\hline Original & Count & Low grade & 21 & 0 & 21 \\
& \multirow{2}{*}{$\%$} & High grade & 0 & 29 & 29 \\
& Low grade & 100 & 0 & 100 \\
& High grade & 0 & 100 & 100 \\
\hline
\end{tabular}

Another important finding is that rCBVt value with a threshold more than 1.7 can differentiate between LGG and HGG and gives $96.8 \%$ sensitivity, $95.2 \%$ specificity, and $96 \%$ diagnostic accuracy. The $96 \%$ specificity reflects a high real negative rate which means that most LGGs have been correctly diagnosed. The relatively high sensitivity means that even though few have been misclassified, most HGGs have been correctly diagnosed. These results reflect those of Fatima et al. [22] who also found that LG and HG could be differentiated from rCBV at the cut-off of 1.33 were $100 \%, 67 \%$, and $90 \%$ respectively.

This study corroborates evidence from previous observations, e.g., Eman et al. [31], Soliman et al. [30], Sparacia et al. [32] who found almost comparable rCBV values with relatively high sensitivity and specificity in the distinction between low- and high-grade gliomas, indicating the reliability of $\mathrm{rCBV}$ in this concern.

Interestingly, within the peritumor region, our results revealed that $\mathrm{rCBVp}$ had the ability to differentiate between LGG and HGG, with a $P$ value of 0.0001 . rCBVp ratios ranging from 0.5 to 3 with a mean of 2.4 for HGG; however, for a low-grade glioma, we observed lower rCBVp ratios ranging from 0.3 to 1 with an average of 0.8 .

These results are consistent with different reports [1517], and further argue that malignant infiltration, with neo-angiogenesis, is expanding to the peritumoral HGG tissues but to a lesser extent in LGG. Therefore, rCBVp can be a useful diagnostic tool in glioma grading.

An rCBVp at cut-off value 1, differentiating low-grade glioma from high-grade glioma with a sensitivity of $87.5 \%$ and a specificity of $100 \%$, further confirms the importance of the application of $\mathrm{rCBVp}$ in this case. The corresponding specificity $(100 \%)$ reflecting a high real negative rate which means that all LGGs were correctly diagnosed. These results are also reported by Soliman et al. [30], which revealed a threshold value more than 0.7 with a sensitivity of $100 \%$, a specificity of $66.7 \%$, a VPP of $88.2 \%$, a NPV of $100 \%$, and an accuracy $90.5 \%$ best distinguish high- and low-grade gliomas. On the other hand, Server et al. [16] also reported this.

The most obvious conclusion emerging from the analysis is that the combination of MRS and MR perfusion with conventional MRI improves the accuracy of the distinction between low- and high-grade gliomas up to $100 \%$. This result was also reported by Zonari $\mathrm{P}$ et al. and $\mathrm{Di}$ Costanzo et al. [8, 28]. 
It should be noted that despite the agreement that rCBV is an incredible tool for determining the grading, there is a slight variation in threshold values between literatures. The heterogeneous nature of the tumors, the MRI scanner, the contrast injection protocol, and the method of analysis are partly responsible for these variations.

It is, therefore, necessary to standardize the technique, the method of analysis, the post-treatment, and, subsequently, the rCBV values, so that the DSC-MRI can be applied realistically in the routine protocol of the MRI of brain tumors. In this regard, the American Society for Functional Neuroradiology (ASFNR) has introduced guidelines and recommendations for optimizing the protocol [33].

\section{Conclusion}

Our results found that intratumoral rCBVt has a significant correlation with the degree of malignancy and makes an appreciable distinction between LGG and HGG. In addition, the evaluation of peritumor rCBVp has played a considerable additional role in this regard. The combination of MR perfusion and SPECT increases the sensitivity, specificity, and diagnostic accuracy (100\%) for recognition of low- and high-grade gliomas.

\section{Abbreviations}

CBV: Cerebral blood volume; DSC: Dynamic susceptibility contrast; FLAIR: Fluid attenuation recovery; FOV: Field of view; rCBVp: Relative cerebral blood volume in the peritumoral area; rCBVt: Relative cerebral blood volume in the tumor; ROI: Region of interest; TE: Echo time; TR: Repetition time

\section{Acknowledgments}

The authors thank all the study participants for their patience and support.

\section{Authors' contributions}

AHA and HIM suggest and develop the research idea, reviewing literature. $\mathrm{ASH}$ and $\mathrm{AH}$ were responsible for data collection and analysis, perform statistical analysis, write and revise the manuscript, prepare MRI cases and perform required measurements, prepare figures and tables. MK was responsible for taking a biopsy and refer to us a histopathology result to compare it with our radiological results. All authors have a major contribution in preparing and editing the manuscript. All authors read and approved the final manuscript.

\section{Funding}

This study was financially supported by Grant office of faculty of medicine, Assiut University, which was responsible for the costs of MRI examination including the contrast material (Magnivest) for all patients only; otherwise, the funder had no role in this study; it means that the funder did not have a role in the study design, data collection and analysis, decision to publish, or preparation of the manuscript.

\section{Availability of data and materials}

The data that support the findings of this study are available from Diagnostic and Interventional Radiology department-Assiut university but restrictions apply to the availability of these data, which were used under license for the current study, and so are not publicly available. Data are however available from the authors upon reasonable request and with permission of the head of Diagnostic and Interventional Radiology department-Assiut university.

\section{Ethics approval and consent to participate}

This study was approved by the Research Ethics Committee of the Faculty of Medicine at Assiut University in Egypt. All patients included in this study gave written informed consent to participate in this research. If the patient was less than 16 years old or unconscious at the time of the study, written informed consent for their participation was given by their parent or legal guardian.

\section{Consent for publication}

All patients included in this research gave written informed consent to publish the data contained within this study. If the patient was less than 16 years old, deceased, or unconscious when consent for publication was requested, written informed consent for the publication of this data was given by their parent or legal guardian.

\section{Competing interests}

The authors declare that they have no competing interests.

\section{Author details}

${ }^{1}$ Department of Diagnostic and Interventional Radiology, Faculty of Medicine, Assiut University, Assiut 71116, Egypt. ${ }^{2}$ Department of Neurosurgery, Faculty of Medicine, Assiut University, Assiut, Egypt.

Received: 8 July 2019 Accepted: 11 November 2019

Published online: 10 December 2019

\section{References}

1. Cha S, et al. Intracranial mass lesions: dynamic contrast-enhanced susceptibility-weighted echo-planar perfusion MR imaging. Radiology. 2002; 223(1):11-29.

2. Lev MH, et al. Glial tumor grading and outcome prediction using dynamic spinecho MR susceptibility mapping compared with conventional contrast-enhanced MR: confounding effect of elevated rCBV of oligodendrogliomas [corrected]. AJNR Am J Neuroradiol. 2004:25(2):214-21.

3. Wetzel SG, et al. Relative cerebral blood volume measurements in intracranial mass lesions: interobserver and intraobserver reproducibility study. Radiology. 2002;224(3):797-803.

4. Wong JC, Provenzale JM, Petrella JR. Perfusion MR imaging of brain neoplasms. AJR Am J Roentgenol. 2000;174(4):1147-57.

5. Barker PB, Bizzi A, De Stefano N, Lin DD, Gullapalli R (2010) Clinical MR spectroscopy: techniques and applications: Cambridge University Press

6. Sjobakk TE, Lundgren S, Kristoffersen A, Singstad T, Svarliaunet AJ, Sonnewald $U$ et al (2006) Clinical $1 \mathrm{H}$ magnetic resonance spectroscopy of brain metastases at 1.5T and 3T. Acta Radiol. 47(5):501-508

7. Caulo M, Panara V, Tortora D, Mattei PA, Briganti C, Pravata E et al (2014) Data-driven grading of brain gliomas: a multiparametric MR imaging study. Radiology. 272(2):494-503

8. Di Costanzo A, Pollice S, Trojsi F, Giannatempo GM, Popolizio T, Canalis L et al (2008) Role of perfusion-weighted imaging at 3 Tesla in the assessment of malignancy of cerebral gliomas. Radiol Med. 113(1):134-143

9. Morita N, Wang S, Chawla S, Poptani H, Melhem ER (2010) Dynamic susceptibility contrast perfusion weighted imaging in grading of nonenhancing astrocytomas. J Magn Reson Imaging. 32(4):803-808

10. Vamvakas A, et al. Imaging biomarker analysis of advanced multiparametric MRI for glioma grading. Physica Medica. 2019;60:188-98.

11. Claes A, Idema AJ, Wesseling P (2007) Diffuse glioma growth: a guerilla war. Acta Neuropathol. 114(5):443-458

12. Blasel S, Jurcoane A, Franz K, Morawe G, Pellikan S, Hattingen E (2010) Elevated peritumoural rCBV values as a mean to differentiate metastases from high-grade gliomas. Acta Neurochir (Wien). 152(11):1893-1899

13. Hakyemez B, Erdogan C, Gokalp G, Dusak A, Parlak M (2010) Solitary metastases and high-grade gliomas: radiological differentiation by morphometric analysis and perfusion-weighted MRI. Clin Radiol. 65(1):15-20

14. Lemercier P, Paz Maya S, Patrie JT, Flors L, Leiva-Salinas C (2014) Gradient of apparent diffusion coefficient values in peritumoral edema helps in differentiation of glioblastoma from solitary metastatic lesions. AJR Am J Roentgenol. 203(1):163-169

15. Akbari H, Macyszyn L, Da X, Wolf RL, Bilello M, Verma R et al (2014) Pattern analysis of dynamic susceptibility contrast-enhanced MR imaging demonstrates peritumoral tissue heterogeneity. Radiology. 273(2):502-510

16. Server A, Orheim TE, Graff BA, Josefsen R, Kumar T, Nakstad PH (2011) Diagnostic examination performance by using microvascular leakage, cerebral blood volume, and blood flow derived from 3-T dynamic susceptibility-weighted contrast-enhanced perfusion MR imaging in the 
differentiation of glioblastoma multiforme and brain metastasis. Neuroradiology. 53(5):319-330

17. Svolos P, Tsolaki E, Kapsalaki E, Theodorou K, Fountas K, Fezoulidis I et al (2013) Investigating brain tumor differentiation with diffusion and perfusion metrics at 3T MRI using pattern recognition techniques. Magn Reson Imaging. 31(9):1567-1577

18. Ostrom QT, Gittleman H, Farah P, Ondracek A, Chen Y, Wolinsky Y et al (2013) CBTRUS statistical report: Primary brain and central nervous system tumors diagnosed in the United States in 2006-2010. Neuro Oncol 15(Suppl 2):ii1-i56

19. Schwartzbaum JA, et al. Epidemiology and molecular pathology of glioma. Nat Clin Pract Neuro. 2006;2(9):494-503.

20. Upadhyay N, Waldman AD. Conventional MRI evaluation of gliomas. $\mathrm{Br}$ Radiol. 2011;84 Spec No 2:S107-11.

21. Fawzy FM, Almassry HN, Ismail AM (2016) Preoperative glioma grading by MR diffusion and MR spectroscopic imaging. Egypt J Radiol Nucl Med. 47(4): 1539-1548

22. de Fatima Vasco Aragao M, Law M, Batista de Almeida D, Fatterpekar G, Delman B, Bader AS et al (2014) Comparison of perfusion, diffusion, and MR spectroscopy between low-grade enhancing pilocytic astrocytomas and high-grade astrocytomas. AJNR Am J Neuroradiol. 35(8):1495-1502

23. Naser RKA, Hassan AAK, Shabana AM, Omar NN (2016) Role of magnetic resonance spectroscopy in grading of primary brain tumors. Egypt J Radiol Nucl Med. 47(2):577-584

24. Kousi E, Tsougos I, Tsolaki E, Fountas KN, Theodorou K, Fezoulidis I et al (2012) Spectroscopic evaluation of glioma grading at 3T: the combined role of short and long TE. ScientificWorldJournal. 2012:546171

25. Liu ZL, Zhou Q, Zeng QS, Li CF, Zhang K (2012) Noninvasive evaluation of cerebral glioma grade by using diffusion-weighted imaging-guided singlevoxel proton magnetic resonance spectroscopy. J Int Med Res. 40(1):76-84

26. Toyooka M, Kimura H, Uematsu H, Kawamura Y, Takeuchi H, Itoh H (2008) Tissue characterization of glioma by proton magnetic resonance spectroscopy and perfusion-weighted magnetic resonance imaging: glioma grading and histological correlation. Clin Imaging. 32(4):251-258

27. Zeng Q, Liu H, Zhang K, Li C, Zhou G (2011) Noninvasive evaluation of cerebral glioma grade by using multivoxel 3D proton MR spectroscopy. Magn Reson Imaging. 29(1):25-31

28. Zonari P, Baraldi P, Crisi G (2007) Multimodal MRI in the characterization of glial neoplasms: the combined role of single-voxel MR spectroscopy, diffusion imaging and echo-planar perfusion imaging. Neuroradiology. 49(10):795-803

29. Kikuchi K, Hiwatashi A, Togao O, Yamashita K, Kamei R, Kitajima M et al (2018) Usefulness of perfusion- and diffusion-weighted imaging to differentiate between pilocytic astrocytomas and high-grade gliomas: a multicenter study in Japan. Neuroradiology. 60(4):391-401

30. Soliman RK, Gamal SA, Essa AA, Othman MH (2018) Preoperative grading of glioma using dynamic susceptibility contrast MRI: relative cerebral blood volume analysis of intra-tumoural and peri-tumoural tissue. Clin Neurol Neurosurg. 167:86-92

31. Geneidi EASH, Habib LA, Chalabi NA, Haschim MH (2016) Potential role of quantitative MRI assessment in differentiating high from low-grade gliomas. Egypt J Radiol Nucl Med. 47(1):243-253

32. Sparacia G, Gadde JA, laia A, Sparacia B, Midiri M (2016) Usefulness of quantitative peritumoural perfusion and proton spectroscopic magnetic resonance imaging evaluation in differentiating brain gliomas from solitary brain metastases. Neuroradiol J. 29(3):160-167

33. Welker K, Boxerman J, Kalnin A, Kaufmann T, Shiroishi M, Wintermark M (2015) ASFNR recommendations for clinical performance of MR dynamic susceptibility contrast perfusion imaging of the brain. AJNR Am J Neuroradiol. 36(6):E41-E51

\section{Publisher's Note}

Springer Nature remains neutral with regard to jurisdictional claims in published maps and institutional affiliations.

\section{Submit your manuscript to a SpringerOpen ${ }^{\circ}$ journal and benefit from:}

- Convenient online submission

- Rigorous peer review

- Open access: articles freely available online

- High visibility within the field

- Retaining the copyright to your article

Submit your next manuscript at $\boldsymbol{\nabla}$ springeropen.com 